\title{
ANALOG COMPUTATION VIA WIRELESS MULTIPLE-ACCESS CHANNELS: UNIVERSALITY AND ROBUSTNESS
}

\author{
Mario Goldenbaum ${ }^{\star}$, Holger Boche $e^{\ddagger}$ and Stawomir Stańczak \\ * Lehrstuhl für Informationstheorie und theoretische Informationstechnik, \\ Technische Universität Berlin, Einsteinufer 25, 10587 Berlin, Germany \\ $\ddagger$ Lehrstuhl für Theoretische Informationstechnik, \\ Technische Universität München, Arcisstraße 21, 80333 München, Germany
}

\begin{abstract}
Recently, it has been shown that the superposition property of wireless multiple-access channels can be exploited to compute functions in sensor networks much more efficiently. By using appropriate pre- and post-processing functions operating on real sensor readings and the superimposed signal received by a fusion center, every function of the measurements is in principle computable by means of the wireless channel in which the pre-processing functions, and therefore the transmitting nodes, do not depend on the function of interest. In this paper we extend these general considerations by examining how robust this kind of universality is against variations in network topology due to nodes that drop out of the network or due to new nodes that connect to the network.
\end{abstract}

Index Terms- Computation over multiple-access channels, representation of functions, wireless sensor networks

\section{INTRODUCTION}

Since the computation of functions of the measurements is one of the most challenging problems in sensor networks [1], it was recently found that the natural superposition property of the wireless multiple-access channel (MAC) can profitably be exploited to compute linear functions much more efficiently [2], [3]. In this context, "efficiency" refers to the need of less wireless resources in comparison to approaches that try to avoid interference by using standard medium-access protocols.

To allow the computation of some nonlinear functions by means of the wireless MAC as well, the authors of [4] proposed a novel analog (i.e., non-digital/uncoded) computation scheme that employs appropriate pre-processing functions, operating on real sensor readings prior to transmissions, and a post-processing function, operating on the real superimposed signal received by a fusion center, to match the resulting overall channel to the nonlinear function of interest.

A natural question that arose in this context was which functions are computable ${ }^{1}$ via an ideal MAC and we found in [5] that the corresponding function space is equal to the space of nomographic functions (see Definition 1). If no additional restrictions

M. Goldenbaum and S. Stańczak were supported in part by the German Research Foundation (DFG) under grant STA 864/3-1 and H. Boche by a start-up fund of Technische Universität München.

${ }^{1}$ To avoid confusion with information-theoretic settings please note that whenever we write in this paper "computable", we exclusively mean analog-computable. are imposed, then in fact every real function of $n$ variables is nomographic and thus in principle computable via the channel, where $n$ denotes the number of active nodes in the network. A surprising observation in [5] was that the corresponding pre-processing functions are completely independent of the function the fusion center aims to evaluate at sensor readings, which results in universal computation schemes consisting of nodes with simple architecture that do not need to be updated if the function of interest changes.

In this paper we extend the work of [5] by analyzing the robustness of the universal computation scheme against variations in network topology. More precisely, after introducing the system model in Section 2, we analyze in Section 3 if the universality property is preserved when nodes drop out of the network (due to for instance failures or battery depletion) or when new sensor nodes join the network.

\subsection{Contributions}

The contributions of the paper are summarized as follows:

- We give a precise definition of universality in the context of analog computations over a MAC and state in Lemma 1 a necessary and sufficient condition on pre-processing functions to ensure this universality.

- Theorem 1 and Observation 2 show that the transmitting sensor nodes (i.e., the pre-processing functions) are still independent of the desired function even if some of the nodes drop out of the network. Thus, the universality is robust (i.e., preserved) under the loss or failure of network nodes.

- Theorem 2 and Observation 3 imply that the above mentioned universality is also preserved if new sensor nodes connect to the network such that it is unnecessary to update already existing nodes.

\subsection{Notational Remarks}

The $k$-times cartesian product $\mathbb{A} \times \cdots \times \mathbb{A}$ of a space $\mathbb{A}$ is written as $\mathbb{A}^{k}$. The natural and real numbers are denoted by $\mathbb{N}, \mathbb{R}$, respectively, and $\mathbb{E}:=[0,1] \subset \mathbb{R}$ defines the unit interval. The identity map on any set $\mathbb{A}$ is described by $\mathrm{id}_{\mathbb{A}}: \mathbb{A} \rightarrow \mathbb{A}$. Furthermore, $\mathcal{F}\left[\mathbb{B}^{\ell}\right]$ denotes the space of every function $g: \mathbb{B}^{\ell} \rightarrow \mathbb{R}$, defined on $\mathbb{B}^{\ell} \subseteq \mathbb{R}^{\ell}, \ell \in \mathbb{N}$. Finally, $\mathcal{N}\left[\mathbb{A}^{k}\right]$ denotes the space of nomographic functions with domain $\mathbb{A}^{k}$ which are defined as follows. 
Definition 1 (Nomographic Functions [5]). Let $\mathbb{A}^{k}, k \geq 2$, be a metric space. Then, every $f \in \mathcal{F}\left[\mathbb{A}^{k}\right]$ for which a representation

$$
f\left(x_{1}, \ldots, x_{k}\right)=\psi\left(\sum_{i=1}^{k} \varphi_{i}\left(x_{i}\right)\right)
$$

exists, with $\psi \in \mathcal{F}[\mathbb{R}]$ and $\varphi_{i} \in \mathcal{F}[\mathbb{A}]$, for all $i=1, \ldots, k$, is called nomographic function.

\section{SYSTEM MODEL}

Consider a wireless sensor network consisting of $n \geq 2$ spatially distributed, simultaneously active nodes and a designated fusion center. The nodes jointly observe a certain physical phenomenon resulting in sensor readings $x_{i} \in \mathbb{E}, i=1, \ldots, n$.

We view a sensor network as a collection of distributed computation devices with the aim of efficiently determining a desired function $f \in \mathcal{F}\left[\mathbb{E}^{n}\right]$ of sensor readings at a fusion center (i.e., $\left.f\left(x_{1}, \ldots, x_{n}\right)\right)$, where a strategy is said to be more efficient if it needs less wireless resources to compute function values with the same precision.

Definition 2 (MAC). Let $s_{i}\left(x_{i}\right) \in \mathbb{R}$ be a transmit signal of node $i$ depending on sensed value $x_{i} \in \mathbb{E}, i=1, \ldots, n$, let $h_{i} \in \mathbb{R}$ be a fading coefficient between node $i$ and the fusion center, and let $n_{i} \in \mathbb{R}$ be the receiver noise. Then, we refer to the standard affine model

$$
\left(x_{1}, \ldots, x_{n}\right) \mapsto \sum_{i=1}^{n} h_{i} s_{i}\left(x_{i}\right)+n
$$

of a wireless multiple-access channel as the $M A C$ and to

$$
\left(x_{1}, \ldots, x_{n}\right) \mapsto \sum_{i=1}^{n} s_{i}\left(x_{i}\right)
$$

as the ideal MAC, respectively.

Equation (3) emphasizes that the natural mathematical operation of a wireless MAC is simply summation which can immediately be used to compute linear desired functions much more efficiently in comparison to orthogonalizing protocols such as timedivision multiple access or carrier-sens multiple access [2], [3]. To allow the computation of nonlinear functions by means of the wireless MAC as well, we employ appropriate pre- and post-processing functions defined as follows.

Definition 3 (Pre-Processing Functions). We define the $n$ real functions $\varphi_{i} \in \mathcal{F}[\mathbb{E}]$, operating on the sensor readings $x_{i} \in \mathbb{E}$ (i.e., $\varphi_{i}\left(x_{i}\right), i=1, \ldots, n$ ), to be the pre-processing functions.

Definition 4 (Post-Processing Function). Let $y \in \mathbb{R}$ be the output of the wireless MAC according to Definition 2. Then, we define the univariate function $\psi \in \mathcal{F}[\mathbb{R}]$, operating on $y$ (i.e., $\psi(y)$ ), to be the post-processing function.

Considering the ideal MAC from Definition 2 in combination with transmit signals $s_{i} \equiv \varphi_{i}\left(x_{i}\right), i=1, \ldots, n$, the pre- and post-processing functions transform the MAC such that the resulting overall channel is in principle able to compute every nomographic function.
Remark 1. Although practical computation schemes suffer from limitations such as power constraints, fading, receiver noise, synchronization issues, we consider in this paper computations over an ideal MAC only to focus on the core of the computation problem and the related questions. The extension to realistic MACs (2) follows along similar lines such as in [4], [6].

\section{UNIVERSALITY AND ROBUSTNESS}

In [5] we have shown that every $f \in \mathcal{F}\left[\mathbb{E}^{n}\right]$ is essentially computable by exploiting the superposition property of a wireless MAC (i.e., mathematically $\mathcal{N}\left[\mathbb{E}^{n}\right] \equiv \mathcal{F}\left[\mathbb{E}^{n}\right]$ ). The surprising fact was that the pre-processing functions are universal and thus independent of the desired function. As a consequence the design of transmitting nodes is independent of the desired function as well.

However, the corresponding proof (see [5, Theorem 2]) is based on a result by Buck in [7], where Bucks construction of universal pre-processing functions depends on the number $n$ of active nodes which implies that transmitting sensors have to be adapted if the network topology changes (i.e., the universality is not robust against modified $n$ ). This in turn would be highly undesired in sensor networks such that we ask in this section what happens in general with the universality if the network topology changes. Hence, we start by precisely defining what is meant by universality during the rest of the paper.

Definition 5 (Universality). We say that analog computation over a MAC is universal if the pre-processing functions $\left\{\varphi_{k}\right\}_{1 \leq k \leq n}$ are universal, that is if they allow to compute every desired function $f\left(x_{1}, \ldots, x_{n}\right) \in \mathcal{F}\left[\mathbb{E}^{n}\right]$.

Universal pre-processing functions are highly desirable as they provide valuable insights into the design of all-purpose sensor nodes that can be universally used for computing every desired function of interest in practical wireless sensor networks.

In preparation for the proofs of this section, the following lemma states a necessary and sufficient condition for $\varphi_{1}, \ldots, \varphi_{n}$ to be universal.

Lemma 1. Let $n \geq 2$ be arbitrary but fixed and let $g: \mathbb{E}^{n} \rightarrow \mathbb{R}_{g}$, $g(\boldsymbol{x}):=g\left(x_{1}, \ldots, x_{n}\right)=\sum_{i=1}^{n} \varphi_{i}\left(x_{i}\right)$, with $\mathbb{R}_{g} \subseteq \mathbb{R}$ denoting the range of $g$. Then, $\left\{\varphi_{i}\right\}_{1 \leq i \leq n}$ are universal pre-processing functions for computing every $f \in \mathcal{F}\left[\mathbb{E}^{n}\right]$ if and only if the function $g$ is bijective.

Proof. The proof is a generalization of an idea from [8].

" $\Leftarrow$ ": Let $\boldsymbol{x}^{(1)}, \boldsymbol{x}^{(2)} \in \mathbb{E}^{n}$, with $\boldsymbol{x}^{(1)} \neq \boldsymbol{x}^{(2)}$. Since $g$ is bijective, it follows that $g\left(\boldsymbol{x}^{(1)}\right) \neq g\left(\boldsymbol{x}^{(2)}\right)$ and from the fact that $\mathbb{E}^{n}$ has the cardinality of the continuum, $\mathbb{R}_{g}$ has the cardinality of the continuum as well.

Now, let $g(\boldsymbol{x})=: x \in \mathbb{R}_{g}$ and $g^{\star}$ be a function such that $g^{\star} \circ g=\operatorname{id}_{\mathbb{E}^{n}}$, that is

$$
g^{\star}: \mathbb{R}_{g} \rightarrow \mathbb{E}^{n}, g^{\star}(x)=\left(\begin{array}{c}
g_{1}^{\star}(x)=x_{1} \\
\vdots \\
g_{n}^{\star}(x)=x_{n}
\end{array}\right) .
$$

Then, we conclude

$$
\begin{aligned}
f\left(x_{1}, \ldots, x_{n}\right) & =f\left(g_{1}^{\star}(x), \ldots, g_{n}^{\star}(x)\right)=\left(f \circ g^{\star}\right)(x) \\
& =\psi(x)=\psi\left(g\left(x_{1}, \ldots, x_{n}\right)\right) \\
& =\psi\left(\sum_{i=1}^{n} \varphi_{i}\left(x_{i}\right)\right), \text { with } \psi:=f \circ g^{\star} .
\end{aligned}
$$




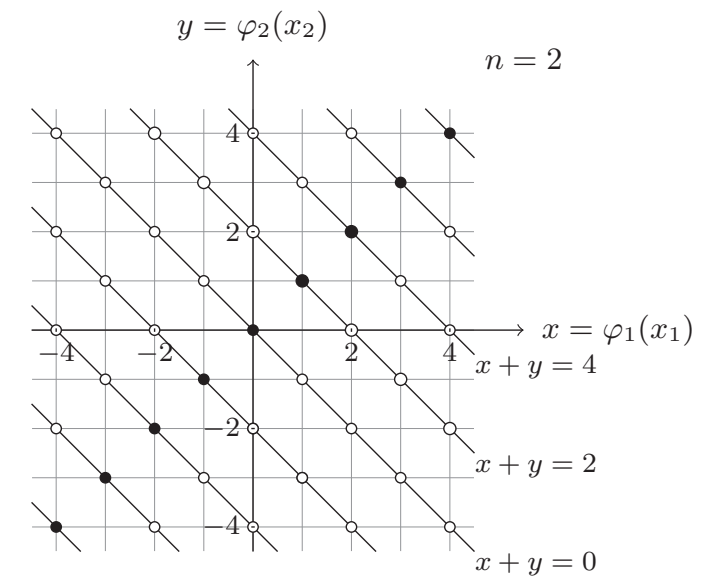

Fig. 1. A line grid of points $g\left(x_{1}, x_{2}\right)=\varphi_{1}\left(x_{1}\right)+\varphi_{2}\left(x_{2}\right)=$ const. to illustrate the bijectivity requirement on function $g$. The preprocessing functions have to be chosen such that $\forall\left(x_{1}^{(1)}, x_{2}^{(1)}\right) \neq$ $\left(x_{1}^{(2)}, x_{2}^{(2)}\right): \varphi_{1}\left(x_{1}^{(1)}\right)+\varphi_{2}\left(x_{2}^{(1)}\right) \neq \varphi_{1}\left(x_{1}^{(2)}\right)+\varphi_{2}\left(x_{2}^{(2)}\right)$. This means in the figure that for example only the black points are allowed in the range of $g$ while the whites have to be avoided.

" $\Rightarrow$ ": If $g$ is not bijective, there exist at least two points $\boldsymbol{x}^{(1)}, \boldsymbol{x}^{(2)} \in \mathbb{E}^{n}$, with $\boldsymbol{x}^{(1)} \neq \boldsymbol{x}^{(2)}$ but $g\left(\boldsymbol{x}^{(1)}\right)=g\left(\boldsymbol{x}^{(2)}\right)$, as well as an $f \in \mathcal{F}\left[\mathbb{E}^{n}\right]$ with $f\left(\boldsymbol{x}^{(1)}\right) \neq f\left(\boldsymbol{x}^{(2)}\right)$. This, however, leads to a contradiction because of $f\left(\boldsymbol{x}^{(1)}\right)=\psi\left(g\left(\boldsymbol{x}^{(1)}\right)\right)=$ $\psi\left(g\left(\boldsymbol{x}^{(2)}\right)\right)=f\left(\boldsymbol{x}^{(2)}\right)$, from which follows that $\left\{\varphi_{i}\right\}_{1 \leq i \leq n}$ are not universal pre-processing functions in the sense of Definition 5 .

Observation 1. For the function $g$ to be bijective, the preprocessing functions $\varphi_{1}, \ldots, \varphi_{n}$ have to be chosen in such a way that for all $\boldsymbol{x}^{(1)}, \boldsymbol{x}^{(2)} \in \mathbb{E}^{n}, \boldsymbol{x}^{(1)} \neq \boldsymbol{x}^{(2)}$, always $\sum_{i} \varphi_{i}\left(x_{i}^{(1)}\right) \neq$ $\sum_{i} \varphi_{i}\left(x_{i}^{(2)}\right)$ holds (see Fig. 1), which means that the ranges of the pre-processing functions have to be appropriate. To illustrate that this is possible, we consider the special case $n=2$ and construct a field $\mathbb{K} \subset \mathbb{R}$ which has the cardinality of the continuum without containing every real number. ${ }^{2}$ More precisely, we consider a real number $\alpha$ which is not in $\mathbb{K}$ (i.e., $\alpha \in \mathbb{R} \backslash \mathbb{K})$ and define $\mathbb{K}$ as the range of $\varphi_{1}$. Furthermore, we define the range of $\varphi_{2}$ to be the field $\frac{1}{\alpha} \mathbb{K}$. Then, for every $\left(x_{1}^{(1)}, x_{2}^{(1)}\right),\left(x_{1}^{(2)}, x_{2}^{(2)}\right) \in \mathbb{E}^{2},\left(x_{1}^{(1)}, x_{2}^{(1)}\right) \neq\left(x_{1}^{(2)}, x_{2}^{(2)}\right)$, it follows $\varphi_{1}\left(x_{1}^{(1)}\right)+\varphi_{2}\left(x_{2}^{(1)}\right) \neq \varphi_{1}\left(x_{1}^{(2)}\right)+\varphi_{2}\left(x_{2}^{(2)}\right)$. Would this not be the case, then

$$
\begin{aligned}
\underbrace{\varphi_{1}\left(x_{1}^{(1)}\right)-\varphi_{1}\left(x_{1}^{(2)}\right)}_{\in \mathbb{K}} & =\varphi_{2}\left(x_{2}^{(2)}\right)-\varphi_{2}\left(x_{2}^{(1)}\right) \\
& =\frac{1}{\alpha} \underbrace{\left(y^{(2)}-y^{(1)}\right)}_{\in \mathbb{K}}
\end{aligned}
$$

would follow and thus $\alpha \in \mathbb{K}$, which would be a contradiction since $\alpha \in \mathbb{R} \backslash \mathbb{K}$.

\footnotetext{
${ }^{2}$ In [9], von Neumann constructed an example of such a field without using the axiom of choice.
}

Remark 2. Lemma 1 and Observation 1 are important because they ensure that such pre-processing functions exist, and therefore open up the possibility of algorithmic constructions.

Remark 3. Note that Lemma 1 and Observation 1 imply that $g$ and $\psi$ are discontinuous functions in general. Since continuity of pre- and post-processing functions can be advantageous for practical implementations, we found in [5, Theorem 3], however, that the requirement for continuous pre- and post-processing functions entails that to compute every continuous desired function of $n$ variables, a sum of $2 n+1$ nomographic functions is necessary. Even if the corresponding $n(2 n+1)$ continuous pre-processing functions are universal as well, the number $2 n+1$ cannot be reduced such that continuity requires more wireless resources.

\subsection{Dropped Out Nodes}

Now let us consider the case where a number of sensor nodes drop out of the network due to for instance failures or battery depletion. The objective is to show that the universality property is preserved when an arbitrary subset of nodes leave the network. To this end, we prove the following theorem.

Theorem 1. The universality of analog computation via an ideal MAC is robust against dropped nodes.

Proof. Let $n \geq 2$ and $\varphi_{1}, \ldots, \varphi_{n} \in \mathcal{F}[\mathbb{E}]$ be fixed universal preprocessing functions to compute every $f\left(x_{1}, \ldots, x_{n}\right) \in \mathcal{F}\left[\mathbb{E}^{n}\right]$. Furthermore, let $\mathcal{I} \neq \varnothing$ be any subset of $\mathcal{I}_{n}:=\{1, \ldots, n\}$. Then, we have to prove that $\left\{\varphi_{k}\right\}_{k \in \mathcal{I}}$ are also universal pre-processing functions to compute every $f\left(x_{1}, \ldots, x_{k}\right) \in \mathcal{F}\left[\mathbb{E}^{k}\right], k \in \mathcal{I}$.

Since the problem is permutation invariant, the numbering of nodes does not matter. Hence, we assume $\mathcal{I}=\mathcal{I}_{\ell}=\{1, \ldots, \ell\}$ with $\ell<n$, since otherwise there is nothing to prove.

If we proceed inductively, we have to show that $\varphi_{1}, \ldots, \varphi_{n-1}$ are universal pre-processing functions for nodes $\{1, \ldots, n-1\}$ to compute every $f\left(x_{1}, \ldots, x_{n-1}\right) \in \mathcal{F}\left[\mathbb{E}^{n-1}\right]$. If this is successful, we arrive in $n-\ell$ steps at $\mathcal{I}_{\ell} \subset \mathcal{I}_{n}$.

We prove the induction hypothesis by contradiction. Assume $\varphi_{1}, \ldots, \varphi_{n-1}$ are not universal pre-processing functions. Then, due to Lemma $1, \sum_{i=1}^{n-1} \varphi_{i}\left(x_{i}\right)$ is not bijective and hence there exist at least two points $\boldsymbol{x}^{(1)}:=\left(x_{1}^{(1)}, \ldots, x_{n-1}^{(1)}\right) \in \mathbb{E}^{n-1}$ and $\boldsymbol{x}^{(2)}:=\left(x_{1}^{(2)}, \ldots, x_{n-1}^{(2)}\right) \in \mathbb{E}^{n-1}, \boldsymbol{x}^{(1)} \neq \boldsymbol{x}^{(2)}$, such that

$$
\sum_{i=1}^{n-1} \varphi_{i}\left(x_{i}^{(1)}\right)=\sum_{i=1}^{n-1} \varphi_{i}\left(x_{i}^{(2)}\right) .
$$

Now we choose an arbitrary $\hat{x}_{n} \in(0,1)$ and consider the points $\hat{\boldsymbol{x}}^{(1)}:=\left(\begin{array}{c}\boldsymbol{x}^{(1)} \\ \hat{x}_{n}\end{array}\right)$ and $\hat{\boldsymbol{x}}^{(2)}:=\left(\begin{array}{c}\boldsymbol{x}^{(2)} \\ \hat{x}_{n}\end{array}\right)$. Of course $\hat{\boldsymbol{x}}^{(1)} \neq$ $\hat{\boldsymbol{x}}^{(2)}$ and therefore

$$
\begin{aligned}
\sum_{i=1}^{n} \varphi_{i}\left(\hat{x}_{i}^{(1)}\right) & =\sum_{i=1}^{n-1} \varphi_{i}\left(x_{i}^{(1)}\right)+\varphi_{n}\left(\hat{x}_{n}\right) \\
& =\sum_{i=1}^{n-1} \varphi_{i}\left(x_{i}^{(2)}\right)+\varphi_{n}\left(\hat{x}_{n}\right)=\sum_{i=1}^{n} \varphi_{i}\left(\hat{x}_{i}^{(2)}\right)
\end{aligned}
$$

contradicts the universality of $\varphi_{1}, \ldots, \varphi_{n}$ when $n$ nodes are active, which proves the preservation of universality for $\mathcal{I}_{n} \rightarrow \mathcal{I}_{n-1}$. Proceeding essentially along the same lines shows that the property is preserved for $\mathcal{I}_{n-1} \rightarrow \mathcal{I}_{n-2} \rightarrow \cdots \rightarrow \mathcal{I}_{2} \rightarrow \mathcal{I}_{1}$. 
Observation 2. It is not necessary to update the remaining active transmitting nodes (i.e., the pre-processing functions) if nodes drop out of the network.

Remark 4. It should be emphasized that even if the pre-processing functions do not depend on the desired function and the number of active nodes, the post-processing function does. This means that the fusion center has to appropriately adapt the post-processing function if the desired function changes or some nodes have dropped out of the network. Furthermore, it has to identify which nodes are inactive.

\subsection{Additional Nodes}

Now we consider the opposite case where an existing sensor network for computation purposes is enlarged by adding a finite number of active nodes. More precisely, assume that we connect $m-n$, $\infty>m>n$, transmitting sensor nodes to the network to universally compute every desired function $f\left(x_{1}, \ldots, x_{n}, \ldots, x_{m}\right)$ of the measurements. Then, we want to answer the question if the universality is preserved if the existing $n$ active nodes were already able to universally compute every $f\left(x_{1}, \ldots, x_{n}\right)$.

Theorem 2. The universality of analog computation via an ideal $M A C$ is robust against a fixed enlargement of the network.

Proof. The proof follows immediately from the proof of Theorem 1 by setting $n=m$ and by considering all subsets $\mathcal{I}_{\ell}=\{1, \ldots, \ell\}$ of $\mathcal{I}_{m}=\{1, \ldots, m\}$, with $n \leq \ell<m$.

Starting with the assumption that $\left\{\varphi_{k}\right\}_{1 \leq k \leq m}$ are fixed universal pre-processing functions to compute every $f \in \mathcal{F}\left[\mathbb{E}^{m}\right]$, the induction arrives in $m-n$ steps at $\ell=n$ such that $\left\{\varphi_{k}\right\}_{1 \leq k \leq \ell}$ are universal pre-processing functions to compute every $f \in \mathcal{F}\left[\mathbb{E}^{\ell}\right]$, for all $n \leq \ell \leq m$.

Observation 3. It is not necessary to update the existing transmitting nodes (i.e., the pre-processing) if the network is enlarged by adding further active transmitting nodes.

Note that the term "fixed" in Theorem 2 as well as the idea of proof refers to the fact that in the current form, the robustness of universality holds if the original network was already designed for $m>n$ nodes but only $n$ nodes are deployed to the measuring field. Then, adding up to $m-n$ nodes during network operation has no impact on the previous $n$ nodes. The more general case in which the original network was designed for at most $n$ nodes but extended to $m$ nodes afterwards is therefore still an open problem.

It should be emphasized, however, that this limits the practical significance of Theorem 2 only marginally since a network for computation purposes can always be designed by virtually choosing $m$ sufficiently large and using only $n$ out of $m$ nodes in practice.

Remark 5. Such as in the case where nodes drop out (cf. Remark 4), unlike the pre-processing functions, the post-processing function has to be updated if new nodes join the network.

\section{CONCLUSIONS}

In the present paper, we considered a sensor network for efficient analog computations over idealized wireless multiple-access channels. Analog systems become again more important since recently it was shown that digital signal processing has in this context some fundamental limits [10], [11].
The main concern of the paper was to analyze the impact of changing network topologies. Therefore, we first considered the case where nodes dropped out of the network due to for instance failures or battery depletion and afterwards we focused on the scenario where an existing sensor network is extended by integrating additional active nodes.

We found that the universality property which ensures the computability of every desired function via the wireless channel on the basis of a fixed set of pre-processing functions, is robust against any change in network topology. As a consequence with high practical relevance for sensor network applications, the transmitting nodes are independent of the desired function the fusion center wants to compute such that they do not need to be updated if nodes leave or enter the network. Therefore, the architecture of corresponding sensor nodes for computation purposes is universal and of reduced complexity, which make them cheap and easy to handle.

\section{REFERENCES}

[1] A. Giridhar and P. R. Kumar, "Toward a theory of in-network computation in wireless sensor networks," IEEE Commun. Mag., vol. 44, no. 4, pp. 98-107, April 2006.

[2] B. Nazer and M. Gastpar, "Computation over multiple-access channels," IEEE Trans. Inf. Theory, vol. 53, no. 10, pp. 3498 3516, October 2007.

[3] S. Stańczak, M. Wiczanowski, and H. Boche, "Distributed utility-based power control: Objectives and algorithms," IEEE Trans. Signal Process., vol. 55, no. 10, pp. 5058-5068, Oct. 2007.

[4] M. Goldenbaum, S. Stańczak, and M. Kaliszan, "On function computation via wireless sensor multiple-access channels," in Proc. IEEE Wireless Communications \& Networking Conference (WCNC), Budapest, Hungary, April 2009.

[5] M. Goldenbaum, H. Boche, and S. Stańczak, "Analyzing the space of functions analog-computable via wireless multipleaccess channels," in Proc. 8th International Symposium on Wireless Communication Systems (ISWCS), Aachen, Germany, Nov. 2011, pp. 779-783.

[6] M. Goldenbaum and S. Stańczak, "Computing functions via simo multiple-access channels: How much channel knowledge is needed?," in Proc. IEEE International Conference on Acoustics, Speech and Signal Processing (ICASSP), Dallas, USA, Mar. 2010, pp. 3394-3397.

[7] R. C. Buck, "Approximate complexity and functional representation,” J. Math. Anal. Appl., vol. 70, pp. 280-298, 1979.

[8] L. Bieberbach, "Operationsbereiche von Funktionen," in Proc. International Congress of Mathematicians (ICM), Zürich, Switzerland, September 1932, vol. I, pp. 162-172.

[9] J. v. Neumann, "Ein System algebraisch unabhängiger Zahlen," Math. Ann., vol. 99, pp. 134-141, 1928.

[10] H. Boche and U. J. Mönich, "Sampling of deterministic signals and systems," IEEE Trans. Signal Process., vol. 59, no. 5, pp. 2101-2111, May 2011.

[11] H. Boche and U. J. Mönich, "Behavior of the quantization operator for bandlimited, nonoversampled signals," IEEE Trans. Inf. Theory, vol. 56, no. 5, pp. 2433-2440, May 2010. 